\title{
Robust Automatic Face Clustering in News Video
}

\author{
Kaneswaran Anantharajah, Simon Denman, Dian Tjondronegoro, Sridha Sridharan, Clinton Fookes \\ Queensland University of Technology (QUT) \\ k.anantharajah,s.denman, dian, s.sridharan, c.fookes@qut.edu.au
}

\begin{abstract}
-
Clustering identities in a video is a useful task to aid in video search, annotation and retrieval, and cast identification. However, reliably clustering faces across multiple videos is challenging task due to variations in the appearance of the faces, as videos are captured in an uncontrolled environment. A person's appearance may vary due to session variations including: lighting and background changes, occlusions, changes in expression and make up.

In this paper we propose the novel Local Total Variability Modelling (Local TVM) approach to cluster faces across a news video corpus; and incorporate this into a novel two stage video clustering system. We first cluster faces within a single video using colour, spatial and temporal cues; after which we use face track modelling and hierarchical agglomerative clustering to cluster faces across the entire corpus. We compare different face recognition approaches within this framework. Experiments on a news video database show that the Local TVM technique is able effectively model the session variation observed in the data, resulting in improved clustering performance, with much greater computational efficiency than other methods.
\end{abstract}

\section{INTRODUCTION}

The identity of people within a video is a key piece of information that can be used to search for, summarize and associate videos. Through knowledge of who is present in a video, various applications including media monitoring to identify the time allocated to a particular person in a news broadcast, or editorial support for journalists to find videos related to a particular person, become possible.

To gather identity information across a video corpus, individuals need to be clustered using a biometric such as face. Reliably clustering faces across multiple videos is a challenging task due to variations in the appearance of the faces, as videos are captured in an uncontrolled environment. A person's appearance may vary between images due to session variations including: lighting changes, background changes, occlusions, changes in expression and make up. Within a single video, cues aside from biometric identity are present that can be used to cluster faces. Within a single news video, footage will often cut between several scenes and people, with the same scene/person revisited several times. Furthermore, the target number of people in either a single video or across a corpus is unknown. Consideration also needs to be given to time constraints, as any approach needs to be able to scale to a large number of videos.

Existing systems tend to rely on heuristics, or simple comparison methods to cluster faces. While significant research has been done in the fields of face recognition $[1,2]$ and clustering within other domains such as audio (i.e. speech diarisation)
[3]; such approaches have not been deployed to cluster faces across a video corpus. Furthermore, existing techniques are typically restricted to clustering within a single video $[4,5]$, or across multiple videos where subject's faces appear with a near-frontal pose in consistent conditions [6].

In this research, we propose a system to cluster faces in broadcast video using the novel Local Total Variability Modelling (TVM) based face recognition approach. We first cluster faces within a single video by using simple cues such as colour, spatial and temporal information; after which we use novel Local TVM face recognition and hierarchical agglomerative clustering (HAC) to cluster faces across the entire corpus. We compare our proposed Local TVM method with six different face recognition approaches using this framework, and show that the Local TVM face clustering approach offers improved performance over the Local GMM-Free Parts (GMM-FP), Local Intersession Variability Modelling (ISV), Probabilistic Linear Discriminant Analysis (PLDA) and TVM approaches. Further, we shows that the TVM clustering system is also orders of magnitude faster when comparing sequences of faces compared to other approaches.

The remainder of the paper is organized as follows. An overview of existing work is presented in Section II; the face clustering framework is explained in Section III. In Section IV, we present the database and evaluation protocols used in this experiment; and in Section V, we present the experimental results. We conclude the paper in Section VI.

\section{EXISTING WORK}

Face clustering frameworks consist of three common steps: face detection, feature extraction, and clustering. First, the faces present in a video are detected, after which features are extracted from the faces, and the similarity between pairs of faces (or face tracks) is computed. Finally, a clustering algorithm is used to merge the detected faces. Various researchers have proposed face clustering systems [4, 5, 7-9] for video, however they are either restricted by assumptions on pose, environment, etc; or they only operate across a single video, rather than a complete corpus.

Pande et al. [4] proposed a method to cluster the faces in a video using a holistic comparison of the face that captured multiple poses, however this approach was limited to clustering within a single video, limiting appearance variations. A similar system was proposed by [7] who used cloth features in addition to facial appearance. However, this approach was also limited by the use of heuristic rules to select a single 
instance of the face to model. Like [4], the system of [7] was only used to cluster faces within a single video.

A framework was proposed to detect individuals appearing unusually across multiple videos in [6]. First, face tracks were formed based on detection results, and following normalisation, images with expression variations, head rotation and noise were automatically removed. After that, HAC was used to cluster the face tracks. This method was evaluated using videos captured in outdoor and indoor environments, where subjects faces appear with a near-frontal pose. However, the evaluation is limited with only 5 of the 90 subjects appearing in multiple videos. Another approach using HAC was proposed in [9] to identify the number of people appearing in an image set. In this method 21 facial landmark points were extracted and each landmark is modeled independently using Probabilistic Linear Discriminant Analysis. Two different clustering methods, an online approach and HAC, were evaluated and it was shown that HAC performed best. However this approach was only evaluated on a still image dataset and performance was not compared with other approaches.

One potential difficulty in using video when compared to still images is the large amount of data. While additional face images may help verification, it also leads to increased computational costs. Anantharajah et al. [8] presented a framework that selects the highest quality frames available in a video to cluster the face. Features including face symmetry, sharpness, contrast and brightness are used to cluster faces. Normalized feature scores are fused and frames with high quality scores are used in a Local Gabor Binary Pattern Histogram Sequence [10] based face clustering system.

The framework of [8] is designed to discard face images with pose, illumination and brightness variations. However, several recent techniques such as Local ISV and total variability modeling (TVM) [2] have been proposed to recognize faces in the presence of such variations. Local ISV [11] seek to explicitly model session variations, allowing it to be compensated for when comparing faces. The use of techniques such as these for face clustering in multi-media offers an interesting avenue to improve clustering performance.

\section{FACE Clustering Framework}

In the proposed framework, faces are initially detected using a Haar cascade based frontal face detector [12]. Faces that appear with a high amount of overlap in successive frames are grouped to form a set of face tracks, after which they are clustered within the video using cues such as scene changes and local appearance (see Section III A). A fixed number of images are selected by sampling uniformly within the face track to represent each face. Then these selected images are modelled using face modelling techniques. In this paper seven face modelling techniques are investigated: Local GMM FreeParts (Local GMM-FP), Local Inter Session Variability modelling (Local ISV), Probabilistic Linear Discriminant Analysis (PLDA) [13], Local Total Variability modelling (Local TVM) and the global counterparts of GMM-FP, ISV and Total Variability modelling (TVM) [2]. Then, the similarity between two face tracks is calculated using one of the above face track modelling method. Finally, HAC is used to merge the face tracks across the video corpus.

\section{A. Clustering Faces within a Single Video}

Within a single video, cues aside from biometric identity are present that can be used to cluster faces. Within a single news video, footage will often cut between several scenes and people, with the same scene/person revisited several times. This is illustrated in Figure 1. Given the nature of news video, which frequently features people talking to camera with little motion in the scene (aside from the people talking), it is possible to use simple colour descriptors to locate scene changes, detect when a scene is revisited, and detect when the same face reappears.

To cluster faces within a single video, a haar cascade is used [12] to detect frontal faces. Faces are tracked between frames based on a simple distance criteria: if the Euclidean distance between the centre of the bounding boxes of faces in consecutive video frames is less than a threshold (20 in the proposed system), the faces are assumed to belong to the same person. The only situation in which this merge will not occur is there is a scene change between the two frames.

Scene changes are detected using a set of histograms to model the scene appearance. The video frame is divided into a grid of overlapping regions $(3 \times 3$ regions with a $50 \%$ overlap between regions) and a histogram is built for each region. Each new frame is compared to the current scene model using the average histogram intersection of the set of scene histograms. If the average intersection, $D$, is less than a threshold $(0.75$ in the proposed system) then the scene is deemed to have changed, otherwise the scene histograms are updated, using

$$
H_{r}^{\prime}{ }^{\text {scene }}(n)=H_{r}^{\text {scene }}(n) \times \alpha+H_{r}^{\text {frame }}(n) \times(1-\alpha) \text {, }
$$

where $H_{r}^{\prime}$ scene $(n)$ is the updated bin value for region $r$ of the scene histogram, $H_{r}^{\text {frame }}(n)$, is the equivalent region and bin in the current frame, and $\alpha$ is a learning rate (set to 0.99). When a new scene is detected, the model of the previous scene is retained to allow for scenes to be later compared when merging faces.

The appearance of a face is modelled using covariance features [14]. Covariance features are used here as there is no need to build the appearance model for a face progressively (i.e. frame by frame), as is done for scenes. To use covariance features for scene detection/modelling would be very computationally expensive as covariance features would need to be recomputed every frame. As with the scene modelling, faces are split into a grid of overlapping regions $(2 \times 2$ regions with a $50 \%$ overlap), and covariance features for the three colour channels are extracted for each region. Covariance features are compared for each region using the method proposed in [15], scores for the regions are averaged to obtain an overall score, after which the similarity is normalised into the range [0..1] (where 1 is a perfect match), 


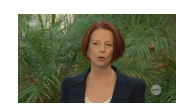

(a) 0:04

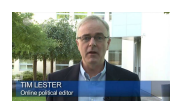

(b) $0: 16$

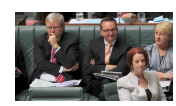

(c) $0: 28$

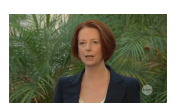

(d) $0: 40$

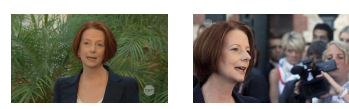

(e) $1: 16$

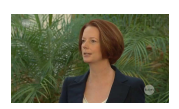

(g) $2: 28$

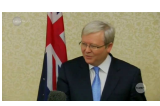

(h) 3:04

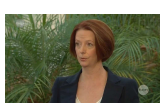

(i) $3: 28$

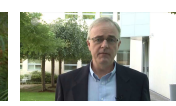

(j) $3: 53$

Fig. 1. Timeline for an example video: Over the course of the video clip, several scenes and people are revisited. The video clip starts with the scene shown in (a) which is revisited in (d)-(e), (g) and (i). Similarly, the location and presenter in (b) is revisited at the end of the clip in (j).

$$
\begin{aligned}
C(i, j) & =1-\frac{\chi(i, j)}{C_{\max }} & \text { if } \quad \chi(i, j)<C_{\max } \\
C(i, j) & =0 & \text { otherwise }
\end{aligned}
$$

where $C(i, j)$ is the similarity between the covariances features belonging to faces $i$ and $j ; \chi(i, j)$ is the similarity computed using [15]; and $C_{\max }$ (set to 5) is a used to normalise the unbounded $\chi(i, j)$ into [0..1].

After a video has been processed and segmented into scenes, faces are merged based on their appearance. A similarity matrix between the faces is constructed as follows:

$$
\begin{aligned}
S(i, j)=0 \text { if }\left\{i_{0}, i_{1}, \ldots i_{p}\right\} \cap\left\{j_{0}, j_{1}, \ldots j_{q}\right\} & \neq \emptyset \\
S(i, j)=C(i, j) & \text { if } i_{\text {scene }}==j_{\text {scene }}
\end{aligned}
$$

$S(i, j)=\sqrt{C(i, j) \times D\left(i_{\text {scene }}, j_{\text {scene }}\right)}$

otherwise,

where $S(i, j)$ is the similarity between two faces, $i$ and $j ;\left\{i_{0}, i_{1}, \ldots i_{p}\right\}$ and $\left\{j_{0}, j_{1}, \ldots j_{q}\right\}$ are the sets of frames that contain faces $i$ and $j ; C(i, j)$ is the distance between two sets of covariance features; $i_{\text {scene }}$ and $j_{\text {scene }}$ are the scenes in which the two faces appear; and $D\left(i_{\text {scene }}, j_{\text {scene }}\right)$ is the distance between the two scene models. This ensures that faces that appear at the same time cannot be merged, faces that occur within the same scene (but different times) are only compared based on the similarity of the face, and all other faces are compared based on both the similarity of the scene and the face. Hierarchical agglomerative clustering is subsequently used to cluster the faces based on the similarity matrix, $S$.

\section{B. Clustering Faces Across a Corpus}

Seven approaches are investigated to compare faces: PLDA [13], Local GMM-FP [11], Local ISV [11], Local TVM and the global counterparts of GMM-FP, ISV and TVM [2].

PLDA [13] has previously been successfully applied to face clustering [9]. We apply this technique as our base line system to model the face tracks. PLDA models an observation as the sum of a mean and offsets that describe the within subject and between subject variation. Interested readers are referred to [9] for more details.

The local GMM-FP and local ISV techniques both divide a face into a set of $R$ regions and model each region independently. DCT features are extracted from overlapping blocks within the region, which are modelled using a GMM. Local ISV extends local GMM-FP by introducing a region specific subspace that captures session variation, allowing it to suppressed when comparing faces. More information can be found in [11].

We propose to extend the region based approach in [11] to total variability modelling (TVM) [2], which we outline in the following subsection.

1) Local Total Variability modelling: Total Variability modelling (TVM) [2] has been successfully applied to face verification. TVM is written in supervector notation as follows,

$$
\boldsymbol{Y}_{i, j}=\boldsymbol{m}+\boldsymbol{T} \boldsymbol{w}_{i, j},
$$

where $\boldsymbol{Y}_{i, j}$ represent the mean supervector of the GMM that best represents the $j$-th image of $i$-th face track. The term $\boldsymbol{m}$ is the mean super-vector of the UBM. The term $T$ is a low rank rectangular matrix and $\boldsymbol{w}_{i, j}$ is the 'i-vector' and represents the total variability.

In this section, a local regions based approach is applied to TVM so that total variability can be locally modelled, allowing the capture of local identity information. A similar concept to that of the Local GMM-FP and Local ISV approaches is applied by dividing the image into $R$ regions and modelling the TVM in each region independently. Thus for the $j$-th image of the $i$-th client in the $r$-th region the model is obtained by,

$$
\boldsymbol{Y}_{r, i, j}=\boldsymbol{m}_{r}+\boldsymbol{T}_{r} \boldsymbol{w}_{r, i, j},
$$

where $\boldsymbol{Y}_{r, i, j}$ represents the mean supervector of the GMM that best represents the $j$-th image of $i$-th face track in the $r$-th region, $\boldsymbol{T}_{r}$ is the region specific rectangular matrix and $\boldsymbol{w}_{r, i, j}$ is the region specific 'i-vector' and represents the region specific total variability. The extraction of region specific i-vectors from a probe image is compared to the region specific subject's model i-vectors using cosine distance scoring. Subsequently, all region specific cosine scores are summed and the score between the face track $i$ and image $t$ is calculated.

2) Comparing Sequences of Faces: Face tracks are modelled using one of the previously mentioned face track modelling methods. After that, the score between two face tracks $i$ and $j$ is calculated as follows for the PLDA, GMM-FP, Local GMM-FP, ISV and Local ISV approaches,

$$
s_{a v}(i, j)=\frac{\sum_{t=1}^{C} h_{\text {score }}\left(\boldsymbol{O}_{t}, \boldsymbol{s}_{i}\right)+\sum_{u=1}^{L} h_{\text {score }}\left(\boldsymbol{O}_{u}, \boldsymbol{s}_{j}\right)}{C+L}
$$

where $s_{a v}$ is the average distance between two face tracks, and $C$ and $L$ are the number of faces selected from face tracks $i$ and $j$ respectively. $h_{\text {score }}\left(\boldsymbol{O}_{t}, \boldsymbol{s}_{i}\right)$ is the similarity score between two face tracks which is the linear score for the local GMM and local ISV as well as their global counterparts; or the LLR for PLDA. The metric is formulated to be symmetric 
(something which neither the LLR or linear score are) as this is required by HAC when clustering faces.

For the TVM and Local TVM approaches each face track is represented by the mean 'i-vector' of the face images. Then the distance between two face tracks is calculated based on cosine distance between the mean ' $\mathrm{i}$-vector' of two face tracks $i$ and $j$.

3) Clustering Faces: In this experiment the HAC with the complete linkage criteria is used to merge the two nearest cluster pairs. The similarity between two observations sets $A$ and $B$, is calculated using complete linkage criteria as below,

$$
s_{c l}=\max \left\{s_{a v}(a, b): a \in A, b \in B\right\},
$$

where, $a$ and $b$ are face clusters.

\section{Database and Evaluation Protocols}

\section{A. News Media Database}

A highly variable news video database $[8,16]$ is used to evaluate the proposed framework. This database consists of 55 news videos with a total length of 123 minutes. In this database, 110 subjects appear, $50 \%$ of whom appear across multiple videos including two subjects who appear in 27 videos. Example images from the database are shown in Figure 2. In this database the subjects identity and face bounding box location at every tenth frames is labeled. Subjects appearing for short periods of time are ignored.

This database has been divided into two subsets: $d e v$ and eval. The dev subset consists of 91, 231 annotated faces from 27 videos and the eval subset consists of 81,812 annotated faces from 28 videos. In the dev subset 98 subjects appear, and in the eval subset 26 subjects appear. In both the dev and the eval subsets 14 subjects appear in common as there are no two approximately equal disjoint subsets. The dev subset is used to tune the system parameters, and the eval subset is used to evaluate the system performance. In this experiment, we used the MOBIO [17] database to train the UBM, Local ISV subspace $\boldsymbol{U}$, PLDA and TVM.

\section{B. Clustering Performance Metrics}

Cluster purity, cluster coverage [18] and attribution error rate (AER) [3] are used to evaluate the system performance. Cluster purity and coverage are obtained by analyzing the dominant identity within each cluster. Cluster purity is the ratio of the total number of correctly clustered faces to the total number of faces in that cluster; and cluster coverage is the best coverage of a subject's face in a single cluster [18]. Average purity and average coverage are calculated as defined in Equations 19 and 20 of [8] respectively.

The attribution error rate (AER) [3] has been used to evaluate speaker attribution systems. The AER is an extension of diarization error rate (DER) [3], and is a time based measurement of incorrectly labeled speaker time compared to the total amount of reference speaker time. We use the AER metric to evaluate the face clustering system. To calculate the AER, reference face annotation labels are concatenated to

\begin{tabular}{lcccc}
\hline \multirow{2}{*}{ System } & \multirow{2}{*}{ Subset } & \multicolumn{3}{c}{ Metrics } \\
\cline { 3 - 5 } & & DER & Coverage & Purity \\
\hline \multirow{2}{*}{ Baseline } & dev & 78.2 & 22.5 & 98.4 \\
& eval & 82.8 & 19.2 & 99.2 \\
Proposed & dev & 32.3 & 70.9 & 98.8 \\
& eval & 26.5 & 77.0 & 98.1 \\
\hline
\end{tabular}

TABLE I

PERFORMANCE OF WITHIN VIDEO FACE CLUSTERING: BASELINE IS A SYSTEM WHICH DOES NOT MERGE FACES WITHIN A VIDEO AND IS THUS THE OUTPUT OF THE FACE TRACKING.

form the reference face identities. This is compared to the face clustering system label, which is obtained by concatenating the attribution labels.

\section{Evaluation of Face Clustering Performance}

Seven face track modelling approaches are investigated for use in clustering: PLDA, Local GMM-FP, Local ISV, Local TVM, and their global counterparts, GMM-FP, ISV and TVM. These are evaluated on the news video database outlined in Section IV. We evaluate two different approaches to cluster faces across multiple videos: by ignoring (Section Section V A) and incorporating (Section V B) within video cues. A brief evaluation of the within video clustering is also presented in Section V-A.

\section{A. Face Clustering Performance within a Single Video}

The within video face clustering system performance is evaluated by averaging the diarisation error rate over each individual video, rather than over the whole corpus. Results are shown in Table I.

We obtained DERs of $32.3 \%$ and $26.5 \%$ on the dev and eval subsets respectively for the proposed approach. We obtain a high purity and low coverage on both dev and eval subsets. High purity indicates that the within video merge technique is not prone to incorrectly merging faces. The greatly increased coverage demonstrates the efficacy of the proposed approach. However, the coverage is limited by the presence of nonfrontal faces which are not detected, and due to the same subject appearing in multiple environments in a clip (although, the approach is not intended to handle this situation).

\section{B. Face Clustering Performance across a Corpus}

1) Ignoring within Video Cues: In this approach the face tracks that result from the face tracking process are taken and merged these using HAC, i.e. the within video clustering technique outlined in Section III-A is not used.

For the GMM-FP, ISV, Local GMM-FP and Local ISV approaches, when extracting the DCT features block size of $12 \times 12$ with $M=44$ has been used. In this experiment UBMs trained with 512 components using the MOBIO [17] database are used. In the ISV and Local ISV approaches a sub-space of 40 components is used. For the TVM approaches (TVM and Local TVM) a subspace of 300 components is used. For the Local GMM-FP and the Local ISV approaches a region 


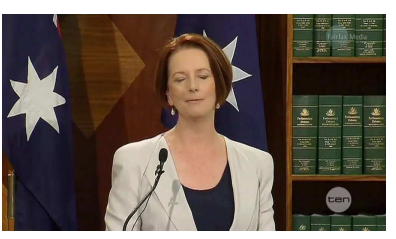

(a)

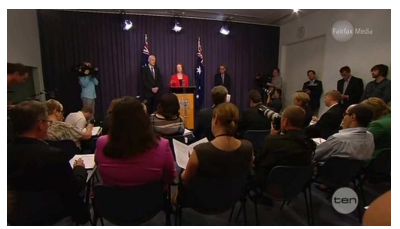

(e)

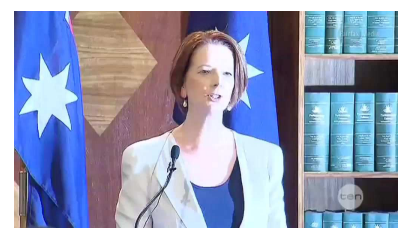

(b)

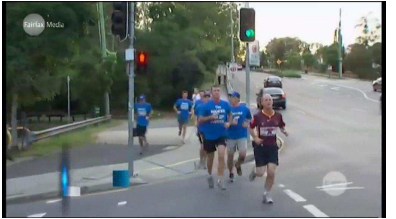

(f)

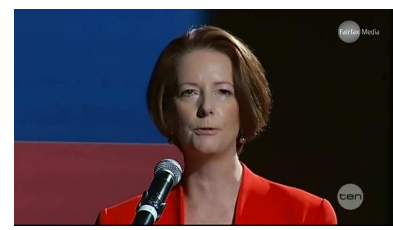

(c)

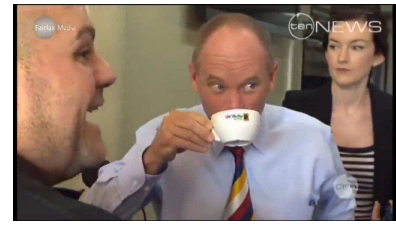

(g)

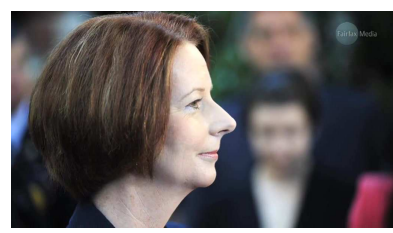

(d)

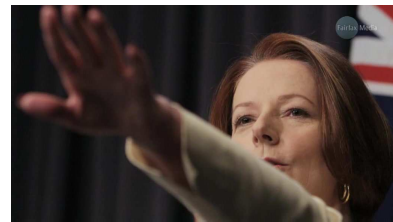

(h)

Fig. 2. Example images show wide variations in illumination (see (a) and (b)), pose (see (c) and (d)), and clutter. Indoor (e) and outdoor (f) footage is present, as are occlusions (see $(\mathrm{g})$ and $(\mathrm{h})$ ). Significant variation in appearance due to the changed imaging conditions (session variation) is evident.

\begin{tabular}{cccccccc}
\hline $\begin{array}{c}\text { Number of } \\
\text { face }\end{array}$ & PLDA & GMM-FP & $\begin{array}{c}\text { Local } \\
\text { GMM-FP }\end{array}$ & ISV & $\begin{array}{c}\text { Local } \\
\text { ISV }\end{array}$ & TVM & $\begin{array}{c}\text { Local } \\
\text { TVM }\end{array}$ \\
\hline 1 & 76.0 & 58.2 & 58.5 & 59.4 & $\mathbf{5 5 . 0}$ & 65.5 & 61.8 \\
2 & 77.4 & 54.8 & 54.7 & 54.2 & $\mathbf{5 2 . 9}$ & 59.2 & 53.6 \\
5 & 75.6 & 52.0 & 53.4 & 53.4 & $\mathbf{5 0 . 8}$ & 57.3 & 51.4 \\
10 & 75.4 & 51.7 & 50.7 & 51.5 & $\mathbf{5 0 . 2}$ & 54.8 & 51.8 \\
20 & 77.1 & 52.7 & 51.9 & 51.8 & $\mathbf{5 0 . 6}$ & 54.8 & 50.9 \\
40 & 75.8 & 52.0 & 52.0 & 51.1 & $\mathbf{5 0 . 1}$ & 55.5 & 51.8 \\
All & 77.7 & 52.7 & 51.9 & 51.7 & $\underline{\mathbf{5 0 . 0}}$ & 55.2 & 51.0 \\
\hline
\end{tabular}

TABLE II

CLUSTERING SYSTEM PERFORMANCE WHEN IGNORING WITHIN VIDEO CUES ON dev SUBSET. THE BEST PERFORMING SYSTEMS ARE SHOWN IN BOLD FOR EACH NUMBER OF SELECTED FACES AND OVERALL BEST PERFORMING SYSTEM IS SHOWN IN BOLD

\begin{tabular}{|c|c|c|c|c|c|c|c|}
\hline $\begin{array}{l}\text { Number of } \\
\text { face }\end{array}$ & $P L D A$ & $G M M-F P$ & $\begin{array}{c}\text { Local } \\
\text { GMM-FP }\end{array}$ & $I S V$ & $\begin{array}{c}\text { Local } \\
\text { ISV }\end{array}$ & $T V M$ & $\begin{array}{l}\text { Local } \\
\text { TVM }\end{array}$ \\
\hline 1 & 78.7 & 72.7 & 67.6 & 71.0 & 67.5 & 71.4 & 66.6 \\
\hline 2 & 79.1 & 66.4 & 63.9 & 64.4 & 58.3 & 71.4 & 62.5 \\
\hline 5 & 73.2 & 57.2 & 62.4 & 57.4 & 54.7 & 63.1 & 59.6 \\
\hline 10 & 73.2 & 55.0 & 58.8 & 57.0 & 59.2 & 61.6 & 59.6 \\
\hline 20 & 73.2 & 57.9 & 58.9 & 58.1 & 55.7 & 61.3 & 55.7 \\
\hline 40 & 73.3 & 58.3 & 58.6 & 55.2 & 55.0 & 64.2 & 55.8 \\
\hline All & 76.9 & 58.1 & 53.6 & 58.4 & $\underline{\mathbf{5 3 . 0}}$ & 63.0 & 54.9 \\
\hline
\end{tabular}

TABLE III

CLUSTERING SYSTEM PERFORMANCE WHEN IGNORING WITHIN VIDEO CUES ON eval SUBSET. THE BEST PERFORMING SYSTEMS ARE SHOWN IN BOLD FOR EACH NUMBER OF SELECTED FACES AND OVERALL BEST PERFORMING SYSTEM IS SHOWN IN BOLD.

size of $2 \times 2$ is used, and for Local TVM a region size of $4 \times 4$ is used. The GMM-FP, ISV and the TVM approaches are a special case of Local GMM-FP, Local ISV and the Local TVM, with a region size of $1 \times 1$ (i.e. a single region such that location information is not considered), and other parameters are the same as the local variants. For the PLDA based approach, normalized grey scale images are modelled using PLDA and a sub-space of 40 components is used. These parameters are tuned using the development subset. In all the approaches all faces, 40, 20, 10, 5, 2 and 1 face images are selected to represent each face by sampling uniformly within the face track.

Tables II and III show the face clustering system performance across the video corpus while ignoring within video cues on the dev and eval subsets respectively. It was found that the Local ISV approach performs best on both the dev and eval sets. The Local ISV system provide an average relative performance improvement of $27.6 \%$ compared to the PLDA approach. It has been observed that local approaches (i.e. Local GMM-FP, Local ISV and Local TVM) outperform their global counterparts on the dev set, but slight degradation is observed for Local GMM-FP on the eval set and this is likely caused caused by face alignment issues.

2) Incorporating within Video Cues: In this approach, face tracks that result from the within video clustering process are taken, such that the input to the between video clustering is a set of longer and more complete face tracks. System parameters and training data are the same as in Section V-B1, 


\begin{tabular}{cccccccc}
\hline $\begin{array}{c}\text { Number of } \\
\text { face }\end{array}$ & PLDA & GMM-FP & $\begin{array}{c}\text { Local } \\
\text { GMM-FP }\end{array}$ & ISV & $\begin{array}{c}\text { Local } \\
\text { ISV }\end{array}$ & TVM & $\begin{array}{c}\text { Local } \\
\text { TVM }\end{array}$ \\
\hline 1 & 56.4 & 53.6 & 50.1 & 50.6 & $\mathbf{4 6 . 7}$ & 53.7 & 47.8 \\
2 & 56.3 & 49.0 & $\mathbf{4 5 . 3}$ & 47.0 & 46.6 & 51.8 & 45.6 \\
5 & 56.7 & 49.0 & 45.2 & 44.1 & $\mathbf{4 3 . 7}$ & 49.1 & $\mathbf{4 3 . 7}$ \\
10 & 56.4 & 46.0 & 44.4 & 44.0 & $\mathbf{4 3 . 9}$ & 47.0 & 44.1 \\
20 & 57.7 & 44.2 & 44.8 & 45.4 & 43.8 & 48.4 & $\mathbf{4 2 . 9}$ \\
40 & 56.4 & 48.5 & $\mathbf{4 3 . 0}$ & 45.5 & 43.9 & 48.5 & 43.8 \\
All & 57.2 & 46.7 & 43.0 & 45.4 & $\underline{\mathbf{4 2 . 8}}$ & 49.4 & 43.1 \\
\hline
\end{tabular}

TABLE IV

CLUSTERING SYSTEM PERFORMANCE BY INCORPORATING WITHIN VIDEO CUES ON dev SUBSET. THE BEST PERFORMING SYSTEMS ARE SHOWN IN BOLD FOR EACH NUMBER OF SELECTED FACES AND OVERALL BEST PERFORMING SYSTEM IS SHOWN IN BOLD.

\begin{tabular}{cccccccc}
\hline $\begin{array}{c}\text { Number of } \\
\text { face }\end{array}$ & PLDA & GMM-FP & $\begin{array}{c}\text { Local } \\
\text { GMM-FP }\end{array}$ & ISV & $\begin{array}{c}\text { Local } \\
\text { ISV }\end{array}$ & TVM & $\begin{array}{c}\text { Local } \\
\text { TVM }\end{array}$ \\
\hline 1 & 68.1 & 65.1 & 64.0 & 61.9 & $\mathbf{5 9 . 3}$ & 61.9 & 61.3 \\
2 & 68.7 & 55.2 & 48.7 & 57.0 & 48.9 & 60.3 & $\mathbf{4 7 . 3}$ \\
5 & 72.9 & 61.0 & 58.2 & 61.1 & 57.2 & 61.8 & $\mathbf{5 3 . 6}$ \\
10 & 72.9 & 59.4 & 49.8 & 53.7 & 48.9 & 62.6 & $\mathbf{4 7 . 5}$ \\
20 & 72.9 & 54.1 & 52.7 & 56.5 & 48.8 & 59.6 & $\mathbf{4 8 . 3}$ \\
40 & 72.9 & 50.2 & 50.5 & 51.7 & 48.8 & 58.3 & $\mathbf{4 7 . 2}$ \\
All & 69.1 & 54.1 & 45.7 & 54.9 & 48.6 & 60.3 & $\underline{\mathbf{4 1 . 0}}$ \\
\hline
\end{tabular}

TABLE V

Clustering SYSTEM PERFoRMANCE BY INCORPORATING WITHIN VIDEO CUES ON eval SUbSET. THE BEST PERFORMING SYSTEMS ARE SHOWN IN BOLD FOR EACH NUMBER OF SELECTED FACES AND OVERALL BEST PERFORMING SYSTEM IS SHOWN IN BOLD.

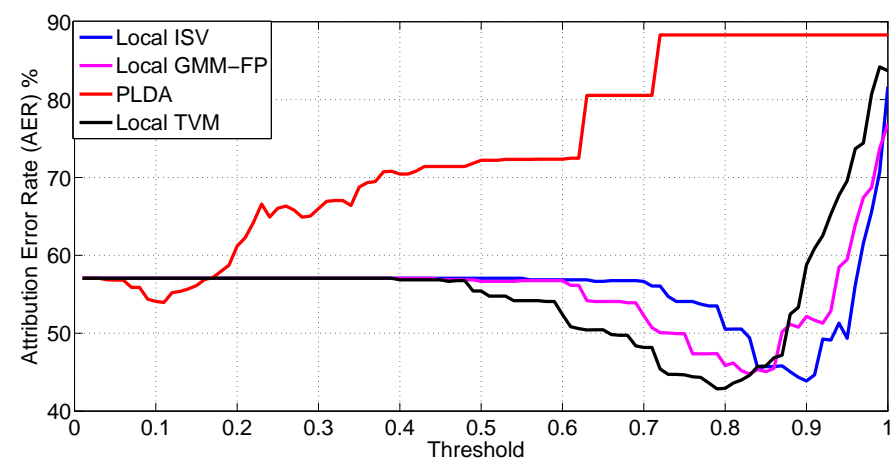

Fig. 3. AER Vs HAC threshold on dev subset when selected faces $=20$

with the exception of the Local GMM-FP and the Local ISV region size, which is now set to $4 \times 4$ as this showed optimum performance on the dev subset. In all the approaches all faces, 40, 20, 10, 5, 2 and 1 face images are selected to represent each face by sampling uniformly within the face track.

Tables IV and V show the face clustering system performance across the video corpus by incorporating within video cues on the dev and eval subsets respectively. It was found that the Local ISV approach performs best on the dev set and the Local TVM approach performs best on the eval set. The Local TVM system provides an average relative performance improvement of $39.8 \%$ compared to the PLDA baseline when all face images are selected to represent the face track. It has been observed that the local approaches outperform their global counterparts with the exception of the GMM-FP method on the dev subset when 20 faces are used to represent face track and on the eval subset when 40 faces are used to represent face track, which performs marginally worse. It has been observed that when the number of faces selected from the face track is increased up to 40 images the GMM-FP based system performance also increases. The higher number of face samples used to enroll and compare faces results in the models better capturing variations in facial appearance, leading to improved performance. This same trend is not observed for the ISV approaches. ISV and Local ISV explicitly models and accounts for session variation, thus reducing the need for a large numbers of faces. The TVM and Local TVM based systems provide better performance as more faces are used to enroll and compare faces, resulting in the models better capturing variations in facial appearance.

By comparing Tables II and IV, it can be seen that for all systems, improvements are achieved on the dev subset. The same trend is observed on the eval subset by comparing Tables III and V. Figure 3 shows the face clustering performance across the news video corpus for the dev set over the HAC threshold when 20 face images are selected to represent the face track. It can clearly be seen that PLDA performs poorly, and predominately merges faces incorrectly as the AER only drops slightly before increasing as more faces are merged. The MOBIO [17] data used to train the PLDA model contains predominately frontal faces, and more pose variation is observed in the news data than is present in MOBIO. As such, the PLDA approach is unable to cope with the large variations observed, and thus performs poorly. The three local approaches perform much better. The Local TVM approach is able to operate well at a wide range of thresholds (i.e. 


\begin{tabular}{cccccccc}
\hline $\begin{array}{c}\text { Number of } \\
\text { face }\end{array}$ & PLDA & GMM-FP & $\begin{array}{c}\text { Local } \\
\text { GMM-FP }\end{array}$ & ISV & $\begin{array}{c}\text { Local } \\
\text { ISV }\end{array}$ & TVM & $\begin{array}{c}\text { Local } \\
\text { TVM }\end{array}$ \\
\hline 1 & 0.2 & 9.9 & 14.7 & 6.8 & 95.8 & 2.1 & 14.2 \\
2 & 0.4 & 19.3 & 27.4 & 7.8 & 96.4 & 3.2 & 16.9 \\
5 & 0.45 & 38.1 & 65.3 & 9.6 & 104.4 & 5.0 & 18.4 \\
10 & 0.62 & 81.2 & 138.5 & 14.3 & 135.6 & 9.3 & 30.4 \\
20 & 1.24 & 181.3 & 274.21 & 30.10 & 201.76 & 19.79 & 54.47 \\
40 & 1.5 & 333.7 & 552.7 & 52.0 & 324.1 & 44.8 & 122.0 \\
All & 8.5 & 2279.9 & 4121.0 & 329.8 & 1829.7 & 333.5 & 734.0 \\
\hline
\end{tabular}

TABLE VI

TIME (CPU SECONDS) TAKEN TO COMPLETE FACE TRACK ENROLMENT ON dev SUBSET.

\begin{tabular}{cccccccc}
\hline $\begin{array}{c}\text { Number of } \\
\text { face }\end{array}$ & PLDA & GMM-FP & $\begin{array}{c}\text { Local } \\
\text { GMM-FP }\end{array}$ & ISV & $\begin{array}{c}\text { Local } \\
\text { ISV }\end{array}$ & TVM & $\begin{array}{c}\text { Local } \\
\text { TVM }\end{array}$ \\
\hline 1 & 0.12 & 1.73 & 2.48 & 1.82 & 4.10 & 0.01 & 0.03 \\
2 & 0.25 & 3.46 & 4.94 & 3.66 & 8.13 & 0.01 & 0.03 \\
5 & 0.57 & 8.50 & 12.23 & 8.98 & 20.07 & 0.01 & 0.04 \\
10 & 1.28 & 16.52 & 24.24 & 17.54 & 39.90 & 0.01 & 0.04 \\
20 & 2.24 & 31.97 & 46.17 & 34.98 & 78.14 & 0.01 & 0.06 \\
40 & 4.64 & 62.71 & 90.24 & 66.59 & 149.10 & 0.01 & 0.06 \\
All & 35.01 & 442.57 & 633.92 & 468.02 & 1049.78 & 0.03 & 0.27 \\
\hline
\end{tabular}

TABLE VII

TIME (CPU SECONDS) TAKEN TO COMPLETE FACE TRACK COMPARISON ON dev SUBSET.

it is fairly stable between 0.75 and 0.85 ), while both the local GMM-FP and local ISV are more sensitive to threshold selection.

Consideration also needs to be given to time constraints, as any approach needs to be able to scale to a large number of videos. Thus, the time taken for the face track enrollment and the face track similarity score calculation is calculated when using different face track modelling techniques. Table VI and Table VII show the average CPU time taken (in seconds) for face track enrollment and comparison steps respectively. The system was implemented in Python and used the freely available signal processing and machine learning tool box, BOB [19]. The experiments were run on a single core of a Intel Xeon E5-2680.

From Table VI it has been observed that the time taken for the face track enrollment for all approaches increase with the number of faces, though TVM and PLDA are quicker than GMM-FP and ISV in all cases. But these are effectively only one-off computations, and comparison time is far more important for clustering. It should also be noted from Table VII that processing times to compare face tracks increase with the number of faces that are extracted from each face track, making the use of a small number of faces preferable. It can be seen that the TVM based clustering system is faster compared to other approaches, particularly for the face comparison. This is due to the use of cosine distance scoring for TVM, while linear scoring is used for Local GMM-FP and Local ISV and the LLR is used for PLDA. The time taken to compare face tracks for the Local TVM based approach is 0.27 second when all face images are used; while for the Local GMM-FP and Local ISV approaches it is 633.92 and 1049.78 respectively. In the $d e v$ subset 218 face tracks are obtained from the within video clustering process. Thus, the distance metric is obtained for 23, 653 possible face track combinations. It should be also noted that when ignoring within video cues 458 face tracks are obtained on the dev subset, increasing the number of possible face track combinations to 104,653 . This clearly demonstrates the necessity for fast face track comparison, and the TVM based approaches are most suitable given this requirement.

We observe that the ISV approach outperforms the TVM approach which is expected given that previous research [2] has shown ISV to perform better for face verification. However, the Local TVM outperforms the Local ISV (except on the dev subset when 1,10 and all faces are selected from the face track) and demonstrates that introducing spatial constraints leads to improved performance for the TVM (Local TVM) based approach. The evaluation presented has used models trained on the MOBIO [17] dataset, with conditions quite different to those observed in the news video data. Although PLDA performed poorly, the Local ISV, GMM-FP and TVM approaches were still able to achieve a moderate level of performance, despite the data mismatch. Previous research [20] has shown that TVM suffers less performance degradation than ISV when confronted with data mismatch between enrollment and testing data. This data mismatch condition which is common in the news video data might contribute to the improved Local TVM face clustering performance.

Overall, experimental results show that face clustering performance can be improved through the use of local methodologies, with Local TVM performing best, due to it's ability to model the session variation that is highly prevalent in news video data. Local TVM is also orders of magnitude faster when comparing sequences of faces compared to ISV, making it a far more practical choice as well. Furthermore, it has been shown 
that incorporating other cues (such as scene and appearance information) can aid performance.

\section{CONClusions AND Future WORK}

In this paper we propose the Local Total Variability Modeling (Local TVM) approach to cluster faces across a news video corpus. A two stage process is proposed to cluster faces: First faces are clustered within a single video using simple cues such as colour and scene changes; and following this face track modelling and HAC is used to cluster faces across the complete corpus. We evaluate both the benefits of the first, within video clustering phase; and the impact of different face recognition approaches and the number of faces used in the second.

We evaluate seven different face recognition approaches: PLDA, Local GMM-FP, Local ISV, Local TVM and their global counterparts of GMM-FP, ISV and TVM. We have shown that Local TVM face clustering yields the best performance. Local TVM provides an average relative improvement of $39.8 \%$ compared to the PLDA baseline. This demonstrates the importance of introducing spatial constraints to TVM, which leads to improvement in the face clustering performance. Furthermore, it has been shown that clustering the faces within a single video using cues such as scene changes and local appearance improves the face clustering performance compared to ignoring this information. The significant speed advantage alongside comparable or better clustering performance compared to ISV makes TVM a strong choice for use in large multi-media systems.

The evaluation presented has used models trained on the MOBIO [17] dataset, with conditions quite different to those observed in the news video data. Although PLDA performed poorly, the Local ISV, GMM-FP and TVM approaches were still able to achieve a moderate level of performance, despite the data mismatch. Future work will look at using alternate data to train models, such as separate news video sources, Multi-PIE [21], or a combination of databases. Another limitation of the proposed approach is the distance measure that is used to compare face tracks, which results in the need to set a detection threshold that varies widely for each system. The use of cross-likelihood ratio [16], widely used in speaker attribution, compares pairs of face tracks to a UBM, such that positive values indicate support for a merge, will be investigated.

\section{ACKNOWLEDGMENT}

This work was supported by the Australian Research Council (ARC) Discovery grant DP140100793.

\section{REFERENCES}

[1] W. Zhao, R. Chellappa, P. J. Phillips, and A. Rosenfeld, "Face recognition: A literature survey," ACM Comput. Surv., vol. 35, no. 4, pp. 399-458, Dec. 2003.

[2] R. Wallace and M. McLaren, "Total variability modelling for face verification," Biometrics, IET, vol. 1, no. 4, pp. 188-199, December 2012.

[3] H. Ghaemmaghami, D. Dean, R. Vogt, and S. Sridharan, "Speaker attribution of multiple telephone conversations using a complete-linkage clustering approach," in Acoustics, Speech and Signal Processing
(ICASSP), 2012 IEEE International Conference on. IEEE, 2012, pp. 4185-4188.

[4] N. Pande, M. Jain, D. Kapil, and P. Guha, "The video face book," in Proceedings of the 18th international conference on Advances in Multimedia Modeling, ser. MMM'12. Berlin, Heidelberg: SpringerVerlag, 2012, pp. 495-506. [Online]. Available: http://dx.doi.org/10. 1007/978-3-642-27355-1_46

[5] S. Foucher and L. Gagnon, "Automatic detection and clustering of actor faces based on spectral clustering techniques," in Computer and Robot Vision, 2007. CRV '07. Fourth Canadian Conference on, may 2007, pp. $113-122$.

[6] J. Barr, K. Bowyer, and P. Flynn, "Detecting questionable observers using face track clustering," in Applications of Computer Vision (WACV), 2011 IEEE Workshop on, jan. 2011, pp. 182 -189.

[7] E. El Khoury, C. Senac, and P. Joly, "Face-and-clothing based people clustering in video content," in Proceedings of the international conference on Multimedia information retrieval, ser. MIR ' 10 . New York, NY, USA: ACM, 2010, pp. 295-304. [Online]. Available: http://doi.acm.org/10.1145/1743384.1743435

[8] K. Anantharajah, S. Denman, D. Tjondronegoro, S. Sridharan, C. Fookes, and X. Guo, "Quality based frame selection for face clustering in news video," in Digital Image Computing: Techniques and Applications (DICTA), 2013 International Conference on, Nov 2013, pp. $1-8$.

[9] S. Prince and J. Elder, "Bayesian identity clustering," in Computer and Robot Vision (CRV), 2010 Canadian Conference on, May 2010, pp. 3239.

[10] W. Zhang, S. Shan, W. Gao, X. Chen, and H. Zhang, "Local gabor binary pattern histogram sequence (lgbphs): a novel non-statistical model for face representation and recognition," in Computer Vision, 2005. ICCV 2005. Tenth IEEE International Conference on, vol. 1, oct. 2005, pp. 786 - 791 Vol. 1.

[11] K. Anantharajah, Z. Ge, C. McCool, S. Denman, C. Fookes, P. Corke, D. Tjondronegoro, and S. Sridharan, "Local inter-session variability modelling for object classification," in Applications of Computer Vision (WACV), 2014 IEEE Winter Conference on, March 2014, pp. 309-316.

[12] P. Viola and M. J. Jones, "Robust real-time face detection," International Journal of Computer Vision, vol. 57, pp. 137154, 2004, 10.1023/B:VISI.0000013087.49260.fb. [Online]. Available: http://dx.doi.org/10.1023/B:VISI.0000013087.49260.fb

[13] P. Li, Y. Fu, U. Mohammed, J. Elder, and S. Prince, "Probabilistic models for inference about identity," Pattern Analysis and Machine Intelligence, IEEE Transactions on, vol. 34, no. 1, pp. $144-157$, jan. 2012.

[14] O. Tuzel, F. Porikli, and P. Meer, "Region covariance: A fast descriptor for detection and classification," in Computer Vision-ECCV 2006. Springer, 2006, pp. 589-600.

[15] W. Förstner and B. Moonen, "A metric for covariance matrices," in Geodesy-The Challenge of the 3rd Millennium. Springer, 2003, pp. 299-309.

[16] H. Ghaemmaghami, D. Dean, and S. Sridharan, "Speaker attribution of australian broadcast news data," in 1st Workshop on Speech, Language and Audio in Multimedia (SLAM2013). Marseille, France: Sun SITE Central Europe, August 2013, pp. 72-77. [Online]. Available: http://eprints.qut.edu.au/63498/

[17] C. McCool, S. Marcel, A. Hadid, M. Pietikainen, P. Matejka, J. Cernocky, N. Poh, J. Kittler, A. Larcher, C. Levy, D. Matrouf, J.-F. Bonastre, P. Tresadern, and T. Cootes, "Bi-modal person recognition on a mobile phone: using mobile phone data," in IEEE ICME Workshop on Hot Topics in Mobile Multimedia, Jul. 2012.

[18] H. Ghaemmaghami, D. Dean, R. Vogt, and S. Sridharan, "Extending the task of diarization to speaker attribution," in Interspeech 2011, Florence, Italy, August 2011. [Online]. Available: http://eprints.qut.edu.au/43351/

[19] A. Anjos, L. E. Shafey, R. Wallace, M. Günther, C. McCool, and S. Marcel, "Bob: a free signal processing and machine learning toolbox for researchers," in 20th ACM Conference on Multimedia Systems (ACMMM), Nara, Japan. ACM Press, Oct. 2012. [Online]. Available: http://publications.idiap.ch/downloads/papers/2012/ Anjos_Bob_ACMMM12.pdf

[20] E. Khoury, L. El-Shafey, C. M. M. Gunther, and S. Marcel, "Bi-modal biometric authentication on mobile phones in challenging conditions," Image and Vision Computing, 2013.

[21] R. Gross, I. Matthews, J. Cohn, T. Kanade, and S. Baker, "Multi-pie," Image Vision Comput., vol. 28, no. 5, pp. 807-813, May 2010. 